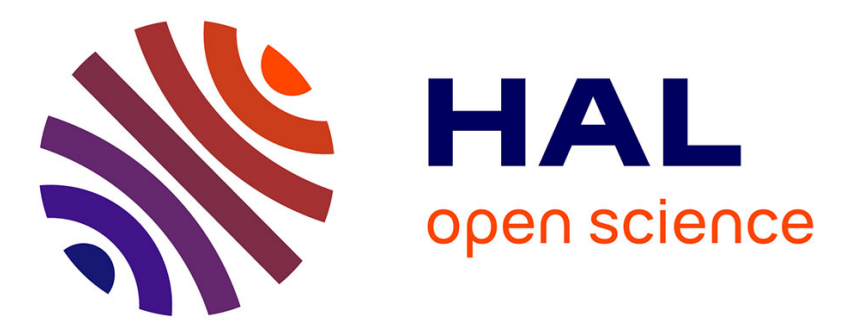

\title{
Contributions to the Design of a 6 DoF Contactless Sensor Intended for Intermittent Contact Haptic Interfaces
}

\author{
Oscar de La Cruz, Florian Gosselin, Wael Bachta, Guillaume Morel
}

\section{- To cite this version:}

Oscar de La Cruz, Florian Gosselin, Wael Bachta, Guillaume Morel. Contributions to the Design of a 6 DoF Contactless Sensor Intended for Intermittent Contact Haptic Interfaces. 2018 3rd International Conference on Advanced Robotics and Mechatronics (ICARM), Jul 2018, Singapore, Singapore. pp.130-135, 10.1109/ICARM.2018.8610825 . hal-02497707

\section{HAL Id: hal-02497707 https://hal.sorbonne-universite.fr/hal-02497707}

Submitted on 3 Mar 2020

HAL is a multi-disciplinary open access archive for the deposit and dissemination of scientific research documents, whether they are published or not. The documents may come from teaching and research institutions in France or abroad, or from public or private research centers.
L'archive ouverte pluridisciplinaire HAL, est destinée au dépôt et à la diffusion de documents scientifiques de niveau recherche, publiés ou non, émanant des établissements d'enseignement et de recherche français ou étrangers, des laboratoires publics ou privés. 


\title{
Contribution to the Design of a 6 DoF Contactless Sensor Intended for Intermittent Contact Haptic Interfaces
}

\author{
Oscar De La Cruz ${ }^{1,2,3,4}$, Florian Gosselin ${ }^{1}$, Wael Bachta ${ }^{2,3,4}$ and Guillaume Morel ${ }^{2,3,4}$
}

\begin{abstract}
The present work presents a theoretical model of an Emitter-Receptor Slit based system (ERS), that uses an infrared light emitting diode (IRED) and a one-dimensional position sensitive detector (PSD), and its experimental validation. The radiation generated by the moving IRED passing through a slit creates a light beam which will form a light spot on the PSD photosensitive area. The PSD generates two photocurrents, function of the amount of received power, which values are used to calculate the position of the light spot center of gravity. The proper response modeling and characterization of the described system will allow the design of a contactless position and orientation sensor, particularly suitable for local tool tracking in force feedback interfaces.
\end{abstract}

Index terms - Robotics, Force feedback interface, Position sensitive detector, Infrared light emitting diode.

\section{INTRODUCTION}

Position measurement technologies are of particular importance in robotics, e.g. encoders installed on a robot manipulator joints provide information about angular displacements which are used in the geometric model of the robot to determine the position and orientation of its endeffector. In this paper, we are more specifically interested in force-feedback systems which are robotic mechanisms capable to measure the user's movements and deliver a force signal to the operator's hand, usually through a pen-like interface, a knob or a thimble. These interfaces usually require the user to be mechanically linked to them. This link has however a nonnegligible influence: the user experiences the friction, inertia and vibrations of the mechanical structure even when moving in free space, which reduces the realism of the interaction. In addition, the difference between free space and contact is less distinctively felt than in real world.

Intermittent-Contact (IC) force feedback interfaces propose to solve this issue by removing the mechanical link between the interface and the user, allowing perfect transparency in free space motion as the user would touch the haptic device only when a contact occurs in the virtual/remote environment. A key component required therefore is an end-effector instrumented with high frequency and precise sensors $(>1 \mathrm{kHz})$ able to calculate the user's hand/tool configuration in space (position and orientation) in order to provide adequate force feedback.

A 2 Degrees of Freedom (DoF) IC force feedback interface has been developed at CEA, LIST [1]. It allows finger

${ }^{1}$ CEA, LIST, Interactive Robotics Laboratory, F-91190 Gif-sur-Yvette, FRANCE.

${ }^{2}$ Sorbonne Université, UPMC Univ Paris 06, Institut des Systèmes Intelligents et de Robotique, F-75005, Paris, FRANCE.

${ }^{3}$ CNRS, UMR 7222, Institut des Systèmes Intelligents et de Robotique, Equipe Agathe, F-75005, Paris, FRANCE.

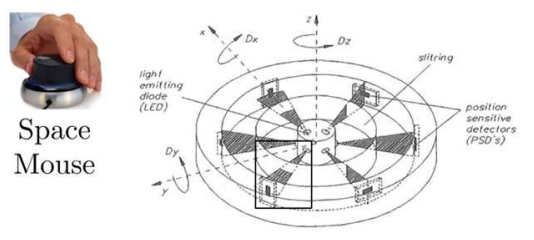

Optical measuring systems
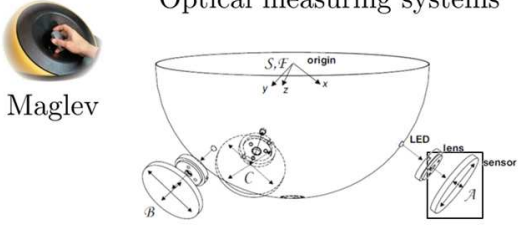

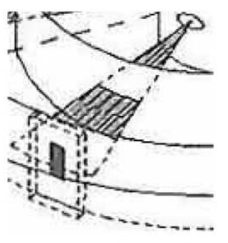

One-dimensional PSD

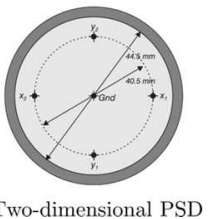

Figure 1. Representative input devices that use PSDs: Space Mouse (top left) [5] and its optical measuring system (top middle and right) [3]. Maglev haptic interface (bottom left) [6], and its optical measurement system [4].

interaction by means of a ring-like end-effector, which is instrumented with infrared proximity sensors. It is used to reconstruct the shape of the finger and precisely estimate its position using distance measurements. Use case applications of this interface remain limited and current end-effector working frequency $(300 \mathrm{~Hz})$ requires to be improved. In order to overcome these limitations, we aim to develop a high frequency $6 \mathrm{DoF}$ end-effector able to provide force feedback by means of a tool, which in contrast with a finger, has the advantage to have a fixed geometry and homogeneous mechanical properties.

A study of available technologies for contactless distance measurement allowed us to identify position sensitive detectors (PSDs) as a suitable option for the development of our endeffector. PSDs are analogical photodetectors used to measure the position of a light spot in either one or two dimensions [2]. When a light spot strikes the PSD photosensitive zone, photocurrents proportional to the light intensity are generated, allowing to determine the incident position. High-speed response, small size and excellent position resolution make PSDs an attractive sensor to our application.

PSDs are commonly used in non-contact distance measurement systems using the triangulation principle for various height and vibration measurements [2]. In robotics we can also find some application cases (see Fig. 1), e.g. the Space Mouse, a 3D-input device used for CAD and robot control, which uses an arrangement of 6 one-dimensional PSDs, slits

${ }^{4}$ INSERM, IU 1150, Institut des Systèmes Intelligents et de Robotique, Equipe Agathe, F-75005, Paris, FRANCE.

\{oscar.delacruzfierro, florian.gosselin\}@cea.fr

\{bachta, Guillaume.morel\}@isir.upmc.fr 
and light emitting diodes (LEDs) to measure the displacement of the handle [3], or the Maglev, a magnetic levitation haptic device [4], which uses three LEDs and three PSDs with lenses providing two-dimension information each, allowing to calculate the flotor's position. Motivated by the versatility of the Emitter-Receptor Slit based systems (ERS) used in the Space Mouse, as well as its potentially low cost, it was chosen as core component to develop our $6 \mathrm{DoF}$ end-effector.

The present document describes the on-going work focused on the modeling and response characterization of an ERS system composed of one infrared light emitting diode (IRED) and one linear PSD. We are particularly interested in predicting the position of the incident light, which will be used to compute the position of the end-effector, as well as the amount of generated photocurrent, whose knowledge is required to ensure that the sensor is not saturated nor under-illuminated, resulting in a poor signal-to-noise ratio. Before describing the proposed method, the essentials of PSDs and radiometry are presented in sections II and III. Section IV details the elements composing an ERS system, section V presents the results and finally conclusions and perspectives are given in section VI.

\section{ONE-DIMENSIONAL POSITION SENSITIVE DETECTOR}

A PSD consists of a uniform resistive layer formed on one or both surfaces of a high-resistivity semiconductor substrate and a pair of output electrodes formed on both ends of the resistive layer for extracting position signals. When a light beam strikes on its photosensitive area, the light incident on the detector is converted into an electrical current (photocurrent) that is divided between the contacts in proportion to the resistance of the active layer (see Fig. 2). This phenomenon is called lateral photo effect [7].

The relation between the location of the incident light and the occurring photocurrents is given by equation (1), where $L x$ is the length of the photosensitive area and $X_{A}$ the distance from the electrical center of the PSD to the light input position, which is independent of the light spot size, shape, and intensity [8].

$$
\frac{I_{X 2}-I_{X 1}}{I_{X 1}+I_{X 2}}=\frac{2 X_{A}}{L x}
$$

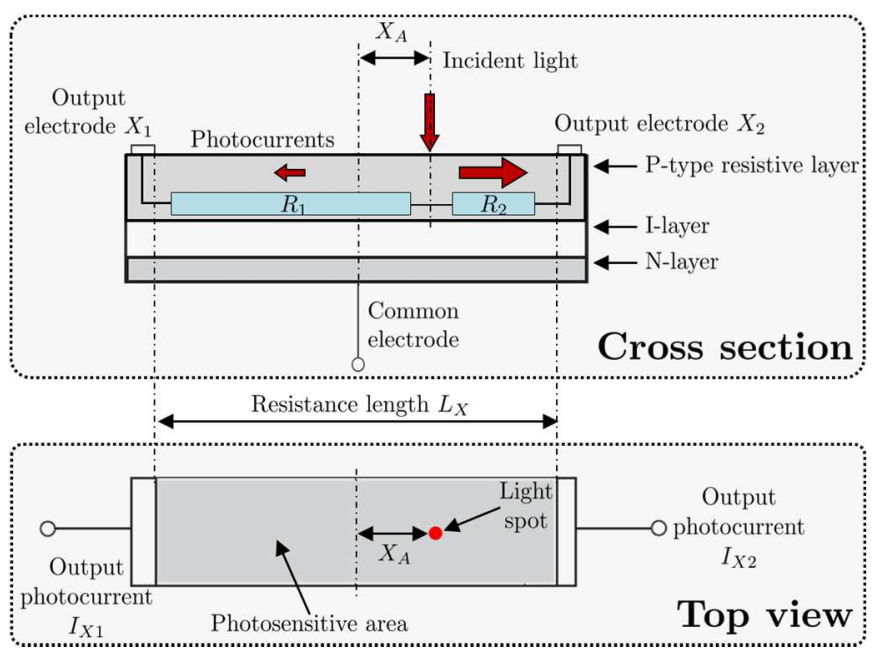

Figure 2. Schematic description of a one-dimensional PSD (adapted from [8]).

\section{BASIC CONCEPTS OF RADIOMETRY}

The spectrum of radiant energy waves that we call light ranges approximately from $380 \mathrm{~nm}$ to $770 \mathrm{~nm}$. Wavelengths shorter (ultra violet) or longer (infrared) than these do not produce a visual response in the eye [9]. In the present article we are interested in the infrared spectrum $(>770 \mathrm{~nm})$, in particular in quantifying the power emitted by an IRED.

Measurement of the propagated energy in electromagnetic waves radiated by light sources uses two approaches: the radiometric approach, which is based on the entire radiant power produced by the source and the photometric approach, which refers only to the part of the radiant power perceived by the human eye as light. Here we deal with the radiometric part.

This study requires the use of solid angles expressed in steradians. The steradian is defined as the solid angle which, having its vertex at the center of a sphere, cuts off a spherical surface area equal to the square of the radius of the sphere (see Fig. 3). The quantity of steradians in a cone is given by equation (2).

$$
\Omega=\frac{A}{r^{2}}
$$

The radiant power or radiant flux $\phi$ represents the total power (light) radiated by a source. Radiant intensity, denoted here as $I_{e}$, refers to the energy radiated by the source into the unit solid angle in a unit of time. This is the power per unit solid angle and is typically given in $\mathrm{mW} / \mathrm{sr}$ by IRED's constructors.

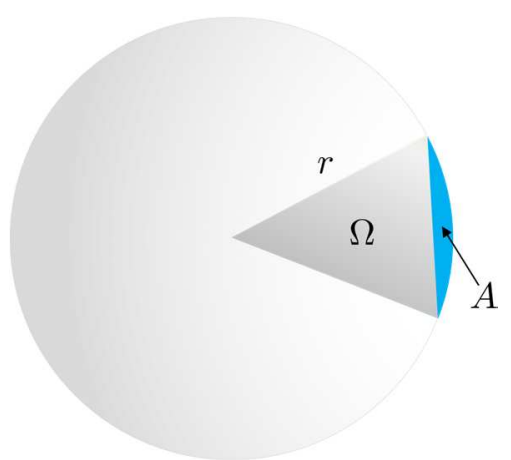

Figure 3. Definition of a solid angle: $\Omega$ represent the amount of steradians contained in the cone, $A$ is the area subtended by the cone and $r$ represents the radius of the sphere.

\section{A. Solid Angle of a Rectangular Plate}

A rectangular aperture is often used in measuring the radiant intensity of a source. For example, the measuring device itself have a rectangular window or sensitive surface [10]. Here we consider a rectangular plate of size $a \times b$ (area $a b)$ at a distance $d$ to an observer, and we consider only the configuration where the vector normal to the plate surface starting at the plate center points to the observer, which means that the plate surface is perpendicular to the line of sight [11]. With the definition of two parameters $\alpha=a /(2 d)$ and $\beta=b /(2 d)$, the solid angle is given by equation (3).

$$
\Omega(a, b, d)=4 \arccos \sqrt{\frac{1+\alpha^{2}+\beta^{2}}{\left(1+\alpha^{2}\right)\left(1+\beta^{2}\right)}}
$$


The presented concepts will be used in the following section to establish a model of the ERS system.

\section{EMITTER-RECEPTOR SLIT BASED SYSTEM}

When a slit is placed in the field of view of a light source, the light passing through it builds a light beam and its shape is mainly determined by the slit geometry. When the light beam hits the photosensitive area of the receptor, a certain amount of power is transmitted to it (see Fig. 4).

The received power does not only depend on the geometry of the light beam but as well on the characteristics of the light source and the irradiated portion of the receptor's photosensitive area. In the following paragraphs we will present the characteristics of the light beam formed by an ERS system that uses one IRED (emitter) and one linear PSD (receptor).

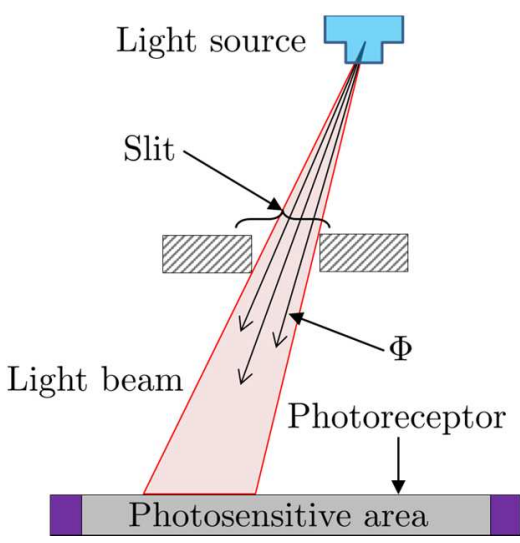

Figure 4. Sagittal view of an ERS system illustrating its main elements.

We are interested here in modeling the response of the ERS system in order to predict two outputs: the position of the light spot $X_{A}$ and the total generated photocurrent $I_{O}=I_{X 1}+I_{X 2}$.

\section{A. IRED-PSD Slit Based System}

The light beam shape generated by this ERS system is similar to a pyramid of rectangular base. As it can be observed in Fig. 5, only a part of its base intersects with the PSD photosensitive area, this intersection represents the light spot $A_{P S D}$. We are interested here in the subsection of the light beam corresponding to this intersection, called here PSD light beam (PLB).

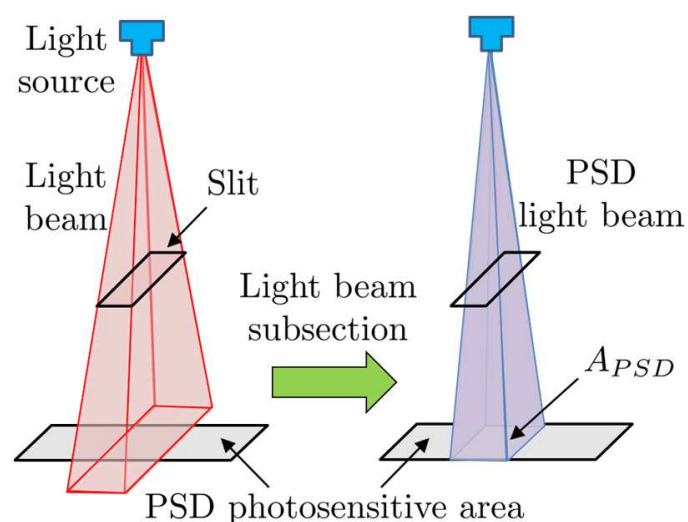

Figure 5. Light beam shaped by the slit (left) and PSD active area (right).
In the present case we suppose that the position of the PLB center of gravity $X_{A}$ corresponds to the geometrical center of the light spot $A_{P S D}$. As the IRED translates in space, the shape of the PLB varies. These variations let us identify other geometrical elements illustrated in Fig. 7.

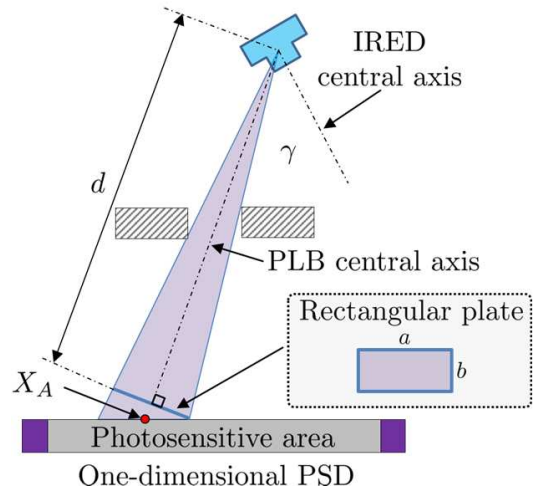

Figure 6. Detailed geometrical description of the PLB. The position of the center of gravity $X_{A}$ does not necessarily intersects with the PSD light beam central axis. The rectangular plate is perpendicular to the PLB central axis and its dimension $a$ is parallel to the photosensitive area.

The amount of solid angle contained in the PLB can be then calculated using equation (3).

\section{B. Photocurrents Calculation}

The generated photocurrent of an isotropic IRED can be calculated using equation (4), where $P_{p s d}$ represents the power received on the PSD photosensitive area, $I_{e}$ is the radiant intensity function of the forward current $I_{F}$ flowing through the IRED and given by the constructor. $S$ represents the photoresponsivity in $\mathrm{A} / \mathrm{W}$, but used here in $\mathrm{mA} / \mathrm{W}$. This is a conversion factor of power into current which is given by the PSD constructor. Finally, $I_{D}$ stands for the dark current.

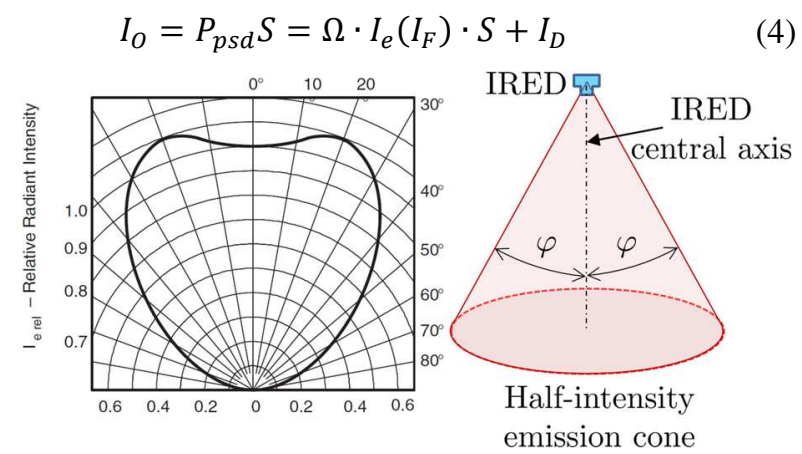

Figure 7. Relative radiant intensity profile for IRED CQY36N (left) and IRED half-intensity emission cone (right). At a view angle $\gamma=\varphi$, the emmitted power corresponds to half of the total radiant intensity $I_{e}$.

IREDs are in practice non-isotropic light sources, this means that depending on the view angle $\gamma$ in reference to the IRED central axis, the emitted power will vary according to a certain profile (see Fig. 7). This variation is regulated by the radiant relative intensity denoted $I_{e R e l}$.

In order to take this power variation into account, we can add the relative radiant intensity $I_{e R e l}(\gamma)$ to equation (4) as follows:

$$
I_{O}=\Omega \cdot I_{e}\left(I_{F}\right) \cdot I_{e R e l}(\gamma) \cdot S+I_{D}
$$


Equation (5) assumes that the relative radiant intensity for a given value of $\gamma$ is representative of the $I_{e R e l}$ distribution inside the solid angle contained in PLB.

In order to ease the calculation of the aforementioned geometrical elements, a simulator of the ERS system has been developed using MatLab (see Fig. 8). The ERS simulator allows to calculate the response of any number of ERS arbitrarily positioned and oriented in space.

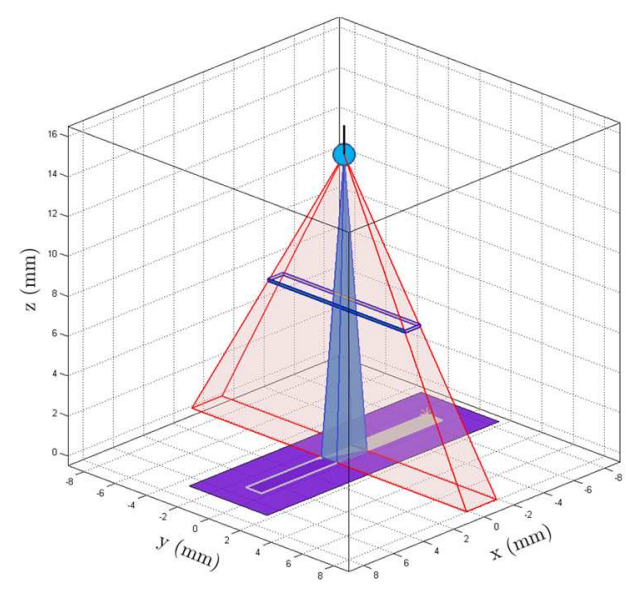

Figure 8. ERS simulator recreating a single ERS system.

\section{RESUlts}

In order to be able to evaluate complex cases, e.g. an arrangement of several ERS systems, the implemented model (see section IV) should provide a response as close as possible to that of the real components. In order to validate the pertinence of the proposed model, a test bench of a single ERS system was build and first experiments were performed. In the following subsections the test bench description, performed experiments and results will be presented.

\section{A. ERS Test Bench}

The test bench is composed of two subassemblies which are denoted here as the Emitter Sub-Assembly (EmSA) and Receptor Sub-Assembly (ReSA). The EmSA can translate in three orthogonal axes, meanwhile the ReSA is static (see Fig. 10).

The ReSA is composed by a receptor support which was specially designed to host the one-dimensional PSD S3932 (see Fig. 10) made by HAMAMATSU. This receptor provides a photosensitive area of $1 \mathrm{~mm} \times 12 \mathrm{~mm}$ and presents a photosensitivity of $S \approx 0.54 \mathrm{~A} / \mathrm{W}$ at a wavelength of $\lambda=$ $950 \mathrm{~nm}$ (corresponding to the IRED wavelength). Its maximal dark current equals $I_{L}=20 \mathrm{nA}$ when the reverse voltage is $V_{R}=5 \mathrm{~V}$.

The EmSA can translate in space thanks to a three orthogonal axes precision translational system (see Fig. 9). The emitter model is CQY36 made by VISHAY. This IRED is particularly interesting because of its big half-intensity emission angle $\varphi= \pm 55^{\circ}$. A big angle allows to increase the space in which the IRED can translate and still send a sufficient amount of radiant power through the slit. The emitter presents a radiant intensity of $I_{e} \approx 3.16 \mathrm{~mW} / \mathrm{sr}$ at a forward current of $I_{F}=100 \mathrm{~mA}$.

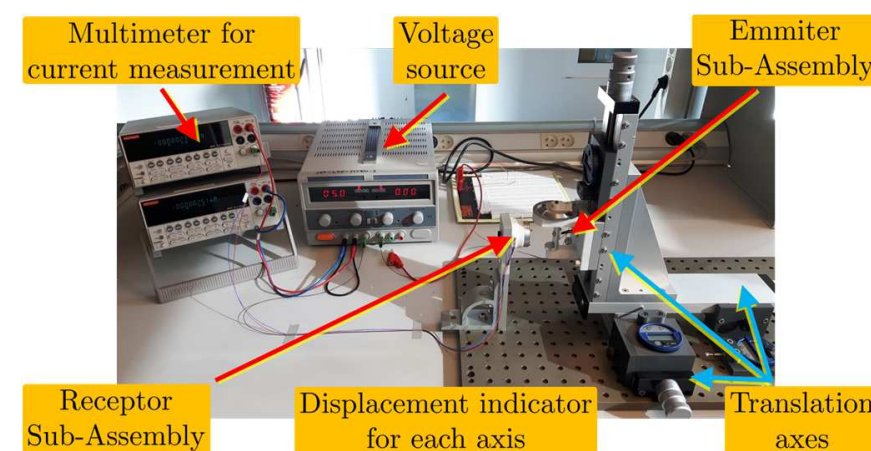

Figure 9. ERS test bench main elements.

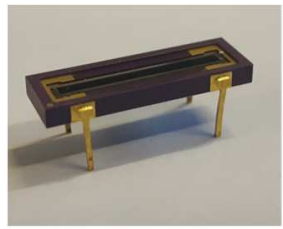

PSD S3932

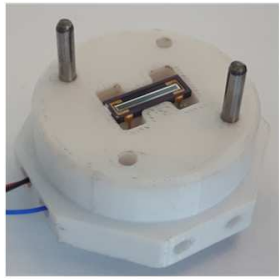

PSD support

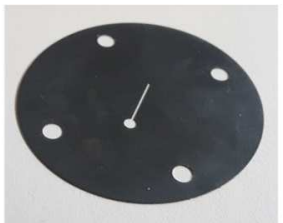

Mask

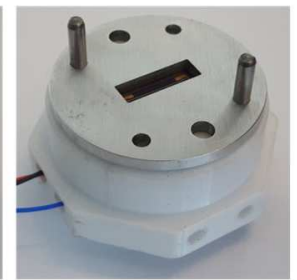

Receptor Sub-Assembly

Shim
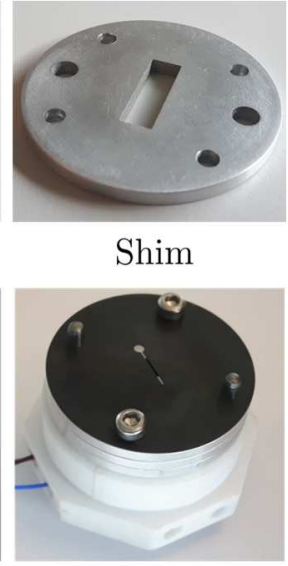

Figure 10. Receptor Sub-Assembly main elements. From left to right, from top to bottom: one-dimensional PSD S3932 by HAMAMATSU provides a photosensitive area of $1 \mathrm{~mm} \times 12 \mathrm{~mm}$, a dark steel mask of $0.15 \mathrm{~mm}$ thick, containing a slit, can be mounted on the ReSA support. The slit width equals $0.4 \mathrm{~mm}$ and it is oriented perpendicular to the PSD photosensitive area, dividing it into two halves. Aluminum shims of $2.5 \mathrm{~mm}$ height allow to position the slit at a distance $d_{B}$ from the PSD surface.
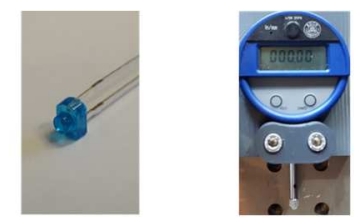

\section{IRED} CQY36N

Displacement
indicator

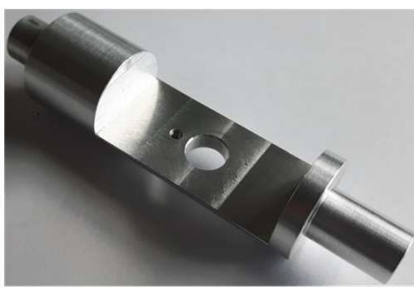

Rotation axis

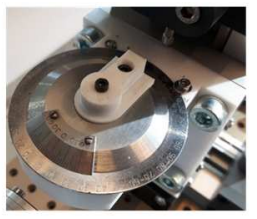

Goniometer

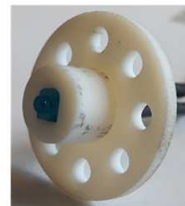

IRED support

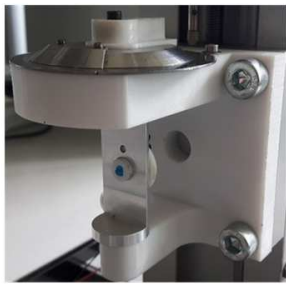

Emitter Sub-Assembly

Figure 11. Emitter Sub-Assembly main elements. From left to right, from top to bottom: IRED CQY36N from Vishay provides a wide half-intensity emission angle $\varphi= \pm 55^{\circ}$, a displacement indicator ROCHPROFIL of $0.01 \mathrm{~mm}$ resolution allows to measure translations on each axis, a goniometer allows to quantify the rotation on the vertical axis, the IRED support can be rotated around IRED's central axis by steps of $45^{\circ}$, the rotation axis hosts the IRED suport and its rotation axis is coincident with the IRED's chip. Finally the complete Emitter Sub-Assembly. 
Both sub-assemblies were mounted parallel to each other on a precision table. Their initial relative positioning is adjusted using a plastic tube of known length that defines a perpendicular distance (of $32 \mathrm{~mm}$ ) between the IRED chip and the PSD surface.
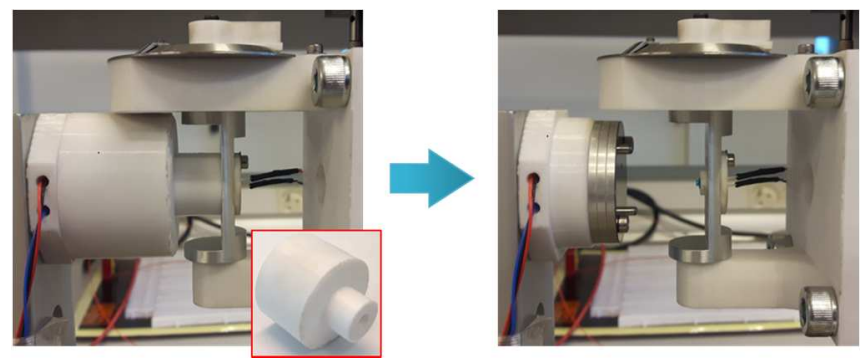

Figure 12. Positioning at the zero reference position by means of the centering tube. At this position the IRED central axis is coincident with the center of the photosensitive area of the PSD.

A shim with an IR filter was designed in order to reduce the pollution due to the visible light (see Fig. 13), however preliminary tests showed that in the current testing environment the visible light had no significant influence on the measured photocurrents. Thus is was not used in the reported experiments.

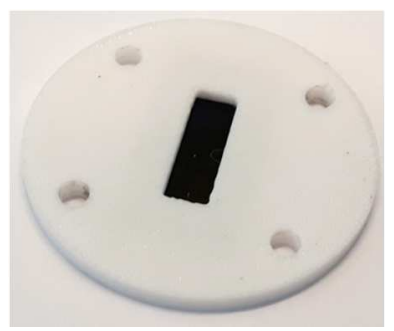

Figure 13. Shim of $2.5 \mathrm{~mm}$ height containing a $1 \mathrm{~mm}$ thick Optolite OM $^{\mathrm{TM}}$ infrared filter made by Instrument Plastics.

\section{B. Experiment Description and Results}

The proposed experiment aims to evaluate the position of the light spot $X_{A}$ and compare the calculated value with the real one. The IRED is translated, with its central axis normal to the PSD surface, along the PSD $x_{P S D}$ axis. The IRED chip was positioned at a distance $d_{A}=15 \mathrm{~mm}$ from the PSD surface and the slit at a distance $d_{B}=7.5 \mathrm{~mm}$ from the same surface. This configuration makes that the displacement of the IRED is almost equivalent to that of the light spot. With a step of $0.5 \mathrm{~mm}$, the displacement interval ranged from -5.5 to 5.5 mm, providing a total of 23 samples of $I_{X 1}$ and $I_{X 2}$. This range guaranties that the light spot remains geometrically inside the photosensitive area.

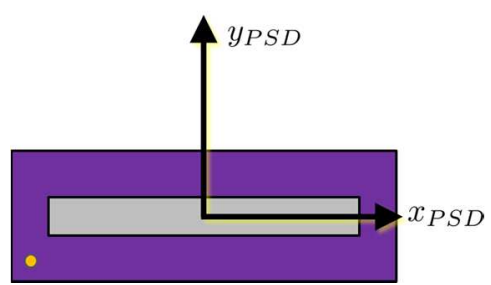

Figure 14. PSD reference frame seen by the IRED. The frame has its origin at the geometrical center of the photosensitive area and the $x_{P S D}$ axis is parallel to it.
Figure 15 shows resulting $X_{A}$ position for the proposed experiment. It can be observed that the calculated and measured values are very similar, however there exists a gap that will be denoted here as absolute error $|\varepsilon|$. It is observed that its value rises as the light spot approaches the extremities of the photosensitive area. The average absolute error is $\widehat{|\varepsilon|} \approx$ $0.2591 \mathrm{~mm}$, the maximum error is $\varepsilon_{\max }=1.1542 \mathrm{~mm}$ and the minimum error is $\varepsilon_{\min }=0.0058 \mathrm{~mm}$.

On the other hand, it can be seen that $|\varepsilon|$ remains low for $-4 \mathrm{~mm}<x_{P S D}<4 \mathrm{~mm}$. The average error here is $\widehat{|\varepsilon|} \approx$ $0.1332 \mathrm{~mm}$. According to [8], the position detection error is measured using a specific part of the photosensitive area. For a PSD which photosensitive area length is $L x \leq 12 \mathrm{~mm}$, the recommended useful area length is only $L x \times 0.75$. This corresponds to a value of $9 \mathrm{~mm}$ for the PSD S3932, i.e. $4.5 \mathrm{~mm}$ on each side of the photosensitive area, which corresponds with the range where $|\varepsilon|$ is low.

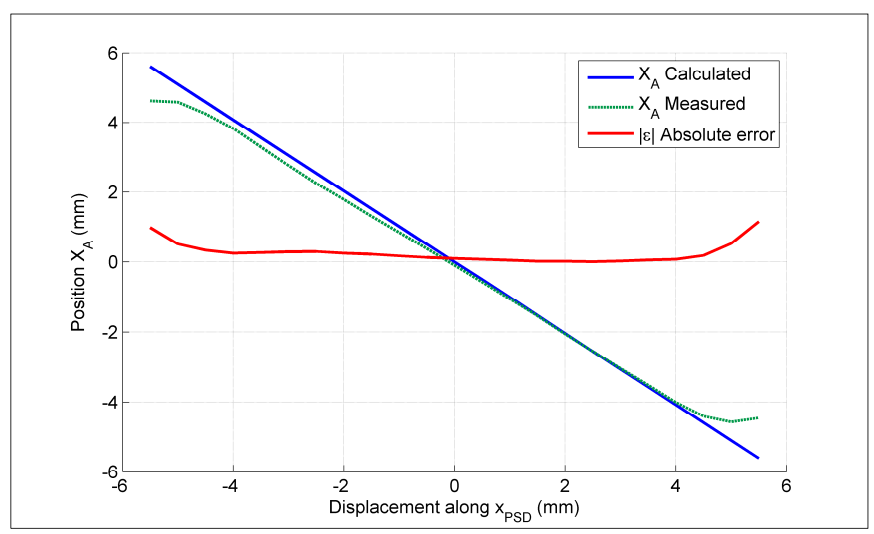

Figure 15. Comparison of calculated and measured values of $X_{A}$. When the IRED translates in the positive direction of $x_{P S D}$, the light spot moves in the opposite direction and vice versa.

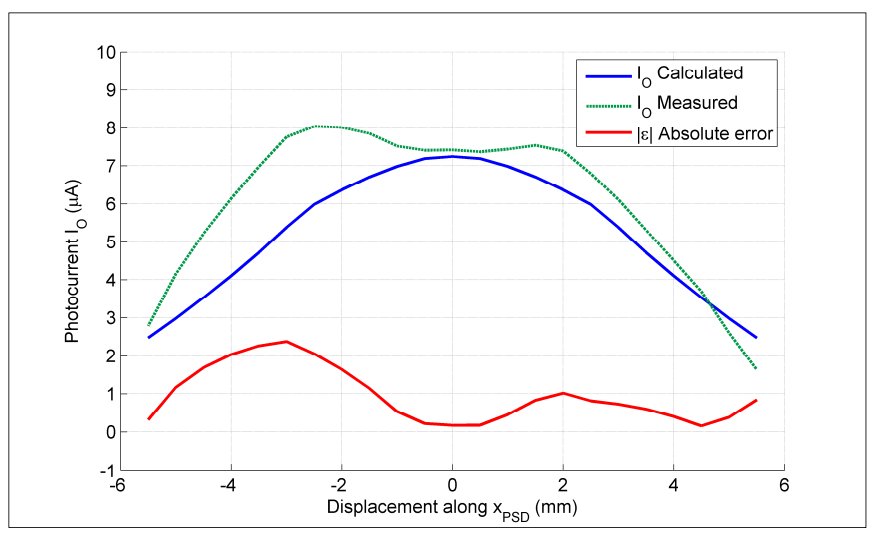

Figure 16. Calculated and measured values of $I_{O}$.

Figure 16 shows the amount of generated photocurrent $I o$ for this experiment. The calculated values of $I_{O}$ preserve a similar order of magnitude in comparison to the measured values. On the other hand the curve profiles are clearly different. A possible explanation is that the theoretical value of $I_{e R e l}$ given in the datasheet differs from that of the real IRED's behavior. In fact, measured $I_{O}$ shows a similar profile to that of the CQY36N radiant relative intensity graph from figure 7 . Due 
to this disparity, a second experiment has been proposed: the IRED rotates around the vertical axis without translating. Initially its central axis is coincident and colinear with the PSD frame origin. The rotation interval ranges from -65 to 65 degrees with an angular step of 5 degrees, providing a total of 27 samples of $I_{X 1}$ and $I_{X 2}$. The positive rotation is defined clockwise when the EmSA is seen from top. The same values for $d_{A}$ and $d_{B}$ used in the previous experiment were kept. The goal of this test was to identify the real radiant relative intensity $I_{\text {ieRel }}$. To do so equation (5) was used and inverted to obtain equation (6) in which $I_{\text {ieRel }}$ is the identified value of the relative radiant intensity and $I_{O m}$ corresponds to the measured photocurrent $\left(I_{D}\right.$ is negligible in practice and was not taken into account). The $I_{\text {ieRel }}(\gamma)$ will be used as a new data instead of $I_{e R e l}(\gamma)$ when calculating $I_{O}$.

$$
I_{i e R e l}(\gamma)=\frac{I_{O m}}{\operatorname{S} . \Omega \cdot I_{e}\left(I_{F}\right)}
$$

Figure 17 shows the obtained result. We can observe that the profile of the calculated total photocurrent $I_{O}$ better fits the measured data. On the other hand, the order of magnitude of the photocurrents has decreased.

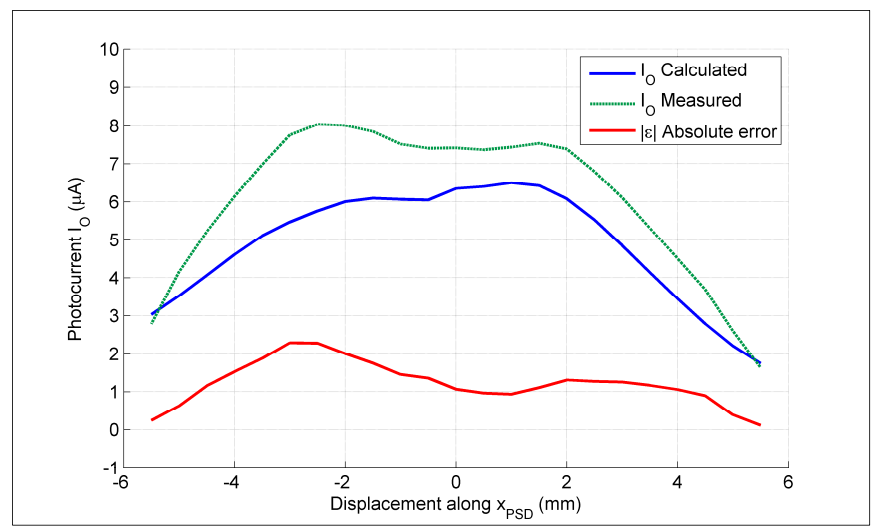

Figure 17. Calculated and measured $I_{O}$ for experiment two.

\section{CONCLUSIONS AND PERSPECTIVES}

The present article introduced a mathematical model of an ERS system intended for the development of a $6 \mathrm{DoF}$ endeffector for IC force feedback interfaces as well as its experimental characterization. Radiometry concepts and the geometrical elements of the ERS system were detailed in order to be able to calculate the amount of radiant flux being received by the photodetector. Elements of conversion of power into photocurrent were also given. A test bench of a single ERS system bas build and tested in a displacement task. The similarity of the calculated position $X_{A}$ with the measured value is particularly promising. The error committed on the estimated photocurrent is still not negligible. If the order of magnitude and profile of the theoretical photocurrents are close to the measurements, it would help to predict an eventual saturation of the receptor or on the contrary to know that for a certain distance there is not enough power received on the receptor. Modeling or characterization require here to be improved.
Characterization of the ERS system is a key point in order to reduce the error margin of simulations of complex cases.

\section{REFERENCES}

[1] Gonzalez, F., Gosselin, F., Bachta, W, “A 2-D Infrared Instrumentation for Close-Range Finger Position Sensing", IEEE Trans. on Instrumentation and Measurement, 64(10), pp. 2708-2719.

[2] Anderson, H., "Position Sensitive Detectors - Device Technology and Applications in Spectroscopy", PhD, Mid Sweden University, Sundsvall, 2008.

[3] Hirzinger, G., "Intuitive Robot Motion Control - The SPACE MOUSE Story", Journal of the Robotics Society of Japan, 17(2), pp. 175-1795(7), 2010.

[4] Berkelman, P. J., "Tool-Based Haptic Interaction with Dynamic Physical Simulations using Lorentz Magnetic Levitation", $\mathrm{PhD}$, Carnegie Mellon University, Pittsburgh, 1999.

[5] 3Dconnexion, https://www.3dconnexion.com/, [March 30, 2018].

[6] Butterfly Haptics, http://butterflyhaptics.com/, [March 30, 2018].

[7] Woltring, H. J., "Single and Dual-Axis Lateral Photodetectors of Rectangular Shape", IEEE Transactions on Electron Devices, 22(8), pp. 581-590, 1975.

[8] Hamamatsu, "Si photodiodes" in Opto-semiconductor handbook, 2014

[9] Ryer, A., Light Leasurement Handbook, Newburyport, MA: Technical Publications Dept., International Light, Inc., 1997, pp. 5-8.

[10] A. Khadjavi, "Calculation of Solid Angle Subtended by Rectangular Apertures", Journal of the Optical Society of America, 58(10), pp. 1417$1418,1968$.

[11] R. J. Mathar, Solid Angle of Rectangular Plate, Technical Note, [Online], Available: http://www2.mpia-hd.mpg.de/ mathar/public/. 